
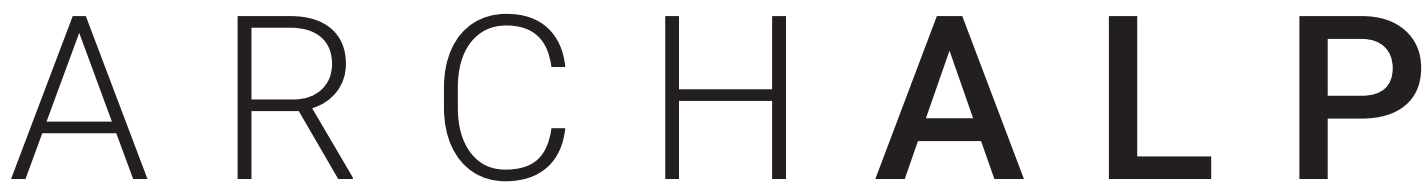

Rivista internazionale di architettura e paesaggio alpino / Revue internationale d'architecture et de paysage dans les Alpes / Internationale Zeitschrift für Alpine Architektur und Landschaft / Revija za alpsko arhitekturo in pokrajino / International journal of alpine architecture and landscape

\title{
Nuove frontiere per il progetto nelle Alpi centrali e orientali
}

De nouvelles frontières pour le projet dans les Alpes centrales et orientales / Neue Grenzen für das Projekt in den Mittel- und Ostalpen / Nove meje projekta $v$ osrednjih in vzhodnih Alpah / New frontiers for the project in the central and eastern Alps 
Rivista internazionale di architettura e paesaggio alpino / Revue internationale d'architecture et de paysage dans les Alpes / Internationale Zeitschrift für Alpine Architektur und Landschaft / Revija za alpsko arhitekturo in pokrajino / International journal of alpine architecture and landscape

Nuova serie / New series n. 05 - 2020

\section{Nuove frontiere per il progetto nelle Alpi centrali e orientali}

De nouvelles frontières pour le projet dans les Alpes centrales et orientales / Neue Grenzen für das Projekt in den Mittel- und Ostalpen / Nove meje projekta v osrednjih in vzhodnih Alpah / New frontiers for the project in the central and eastern Alps 


\section{Indice dei contenuti Contents}

\section{Temi}

Ascoltare il territorio / Listening to the territory opportunity: rethinking construction Daniel A. Walser

\section{Esperienze}

Zwischen regionalem und persönlichem Kontext: die Arbeit von Bernardo Bader / Between regional and personal context: the work of Bernardo Bader

Verena Konrad

Zeitlose Architekturen / Timeless architectures

Markus Wespi, Jérôme de Meuron, Luca Romeo

Progettare con il Genius loci / Designing with the genius loci Nicola Baserga

Vsak projekt je lahko priložnost za krepitev skupnosti /

Meta Kutin

Zwei Bauten, am Berg und im Tal / Two buildings, one in the mountains and one in the valley Andreas Flora 
Progettare in montagna / Designing in the mountains

Gerhard Mahlknecht

Tessere "inattese" in un vecchio mosaico / "Unexpected" tiles

in an old mosaic

Enrico Scaramellini

Tradurre la tradizione / Translating tradition

Federico Mentil

Conoscere i luoghi, interpretare il cambiamento / Knowing places, interpreting change

Alberto Winterle

Ragioni del passato e condizioni del presente / Past reasons and present conditions

Roberto Paoli 
simone cola/daniel walser/ narcus wespi/jerone de nicola baserga/meta kutin scagnol/gerd bergmeister mahlknecht/enrico scaran 
Verena konrad

leuron lica romeo

andreas flora matied

michaela wolf gernard

ellini/federico mentil/

1. TEMI 


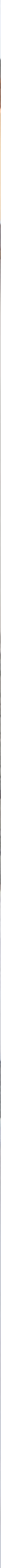




\title{
Architektur als Chance. Bauen neu denken
}

\author{
Architecture as an opportunity: rethinking construction
}

\begin{abstract}
In the Alpine valleys, life is under pressure. Since very few children live in these areas, schools in small towns such as Vrin in the Lumnezia valley (Grisons, Switzerland) are closing, and infrastructures and public life are more and more concentrated in larger centres. Moreover, communities behave differently nowadays: people live and move around the whole valley, and old villages are becoming residential areas. While most of the time architecture in urban areas is driven by the investors' interest, things are different in the mountains. In the Alps, where statistics and the market are not leading criteria for constructing new buildings, architects can explore new directions and think about innovative and specific solutions that may accompany the development of living societies. The recent works by Gion A. Caminada in Valendas and Almens, the buildings by Capaul \& Blumenthal or the architecture projects by Men Duri Arquint in Chur are but a few examples of a different way of looking at the opportunities that architecture may offer to Alpine communities.
\end{abstract}

\section{Daniel A. Walser}

Architect and Professor of Architecture History, Theory and Urbanism at the University of Applied Sciences of the Grisons FHGR in Chur, Switzerland. In his research, he focuses on contemporary architecture in the Alpine regions; he has published works about his research outcomes and curated exhibitions.

\section{Keywords}

Alps, Switzerland, valley, community, society. 
Architekten reagiren auf den gesellschaftlichen Wandel und schaffen neue Lösungen. Gerade im alpinen Raum ist dies essentiell.

In den Alpen sind derzeit grosse Umwälzungen im Gange. Die Abwanderung hat in den entlegenen Tälern in der Schweiz wieder zugenommen. Gelichzeitig sind zentralere Orte gewachsen und übernehmen weitere Zentrumsfunktionen. Dies ist eine Folge des gesellschaftlichen Wandels, wo beispielsweise in Familien beide arbeiten und die Nähe zu Infrastrukturen wie Schulen und Einkaufen wichtiger wird. So wurde beispielsweise in der hintersten Gemeinde des Val Lumnezia in Vrin die Schule geschlossen. Die Schüler reisen nach Vella oder noch grössere Zentren. Meist stehen die Schulhäuser nun leer und werden nicht mehr gebraucht. Damit das in Vrin nicht passiert hat der Architekt Gion A. Caminada sein Architekturbüro in den Bau verlegt. Der gesellschaftliche Wandel verlangt heute nach neuen Lösungen.

Neue Ansätze entwickelte Caminada in der kürzlich fertiggestellten Siedlung «Burggarta und Erlihuus» in Valendas (2020), welche eine Art Schlusspunkt des baulichen Engagements der Stiftung Valendas Impuls ist. Die Stiftung startete das Projekt, um ihr Stiftungskapital so anzulegen, dass sie permanent Einnahmen erhält und so im Dorf langfristig aktiv sein kann. Sie fördert seit Jahren die Reaktivierung des Dorfes mit intelligenten Projekten wie unter anderem dem «Gasthaus am Brunnen» (2014) als Dorfrestaurant mit Gourmetküche für Auswärtige und Hotel von Caminada. Dazu kommt die radikale Restaurierung des Türalihuses von Capaul Blumenthal (2014) für die Stiftung Ferien im Baudenkmal und den Umbau des alten Schulhauses zum Besucherzentrum des nahen Naturparks Beverin von Nickisch Walder (2016). Alle Projekte trugen dazu bei, dass das Dorf heute keine Abwanderung mehr hat und die leeren Häuser wieder instand gestellt und bewohnt werden. Ziel

Vorherige Seite Kunstmuseum Muzeum Susch, Chasper Schmidlin und Lukas Voellmy.

Susch (CH) 2018

(foto Conradin Frei).
Die Siedlung «Burggarta und Erlihuus» ist ein aktiver Beitrag zum Dorfleben am Rand von Valendas mit je einem Gebäude für hochwertige Wohnräume und einem mit Ateliers. Der Komplex

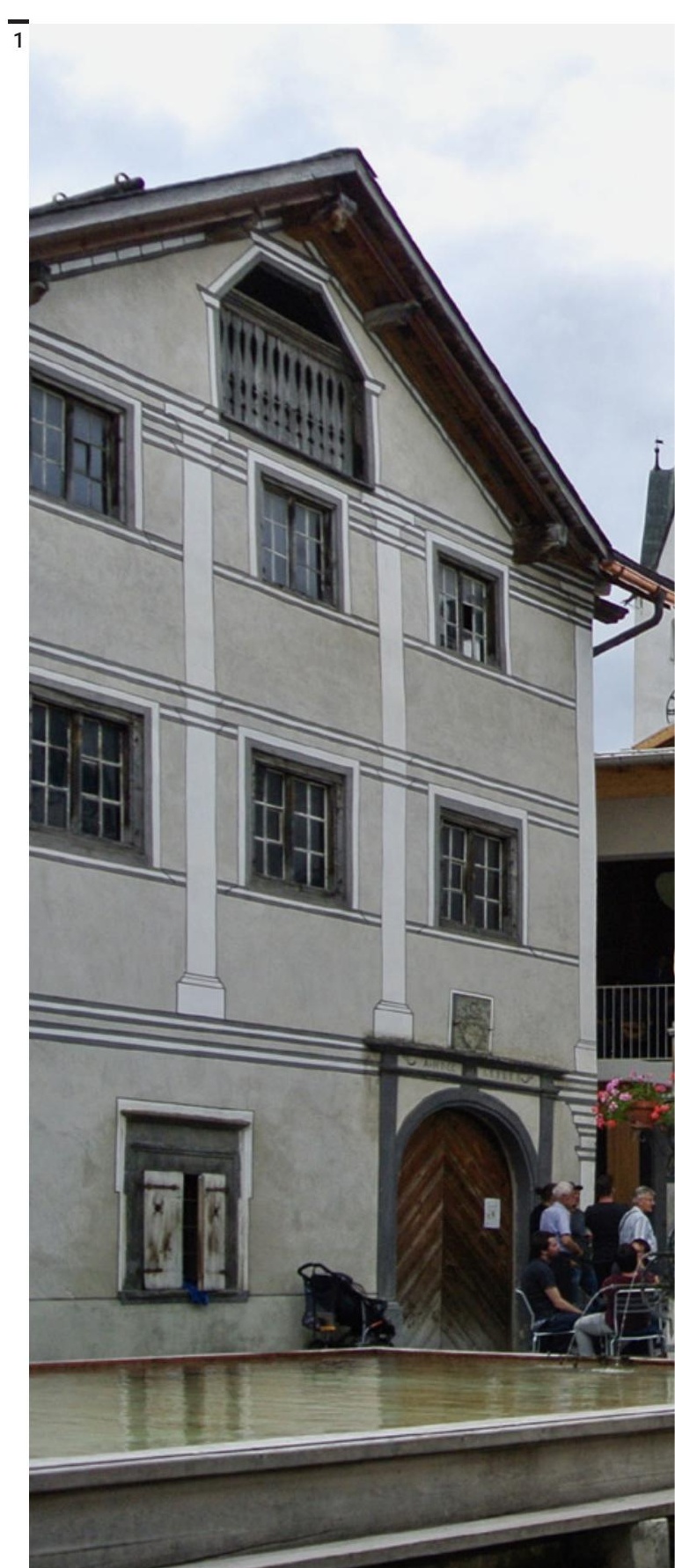


«Burggarta» besitzt sieben Laubenwohnungen für permanente Mieter, welche hier auch ihren Hauptwohnsitz haben. Die Wohnungen besitzen verschieden Klimazonen: beheizte, ungeheiz-

Abb. 1

Dorfplatz mit dem grössten hölzernen

Dorfbrunnen in

Valendas mit

«Gasthaus am Brunnen» (2014) von

Gion A. Caminada (links) und Türalihus (2014) von Capaul Blumental (rechts). Valendas, $\mathrm{CH}$ (foto Daniel A. Walser) täten. Der gemeinsame Innenhof stärkt den Ge- plex im «Erlihuus» einen Raum, der auch externen gemietet werden kann für Anlässe und Treffen aller Art und Werkräume für verschiedenste handwerkliche oder künstlerische Aktivi- meinschaftssinn im Haus. Der Bau versteht sich explizit als integraler Teil des Dorfes, aber auch des Tals. Autos und öffentlicher Verkehr sind fester Bestandteil und nehmen auch baulich einen prominenten Platz ein.

Eine Stufe weiter und vor allem auch grösser geht Gion A. Caminada mit seinem Projekt für die Wohngenossenschaft Pumera in Almens. Der Bau beherbergt 25 verschiedenen Wohnungen, verteilt auf drei bewohnten Baukörpern um einen Innenhof. Der erwartete Baubeginn ist 2021. Auch hier gibt es drei verschiedene Klimazonen wie in Valendas. Das Wohnen organisiert sich um eine gemeinschaftliche, zentrale Küche. Verkehrsflächen, Eingang und andere Zonen sind explizit unbe-

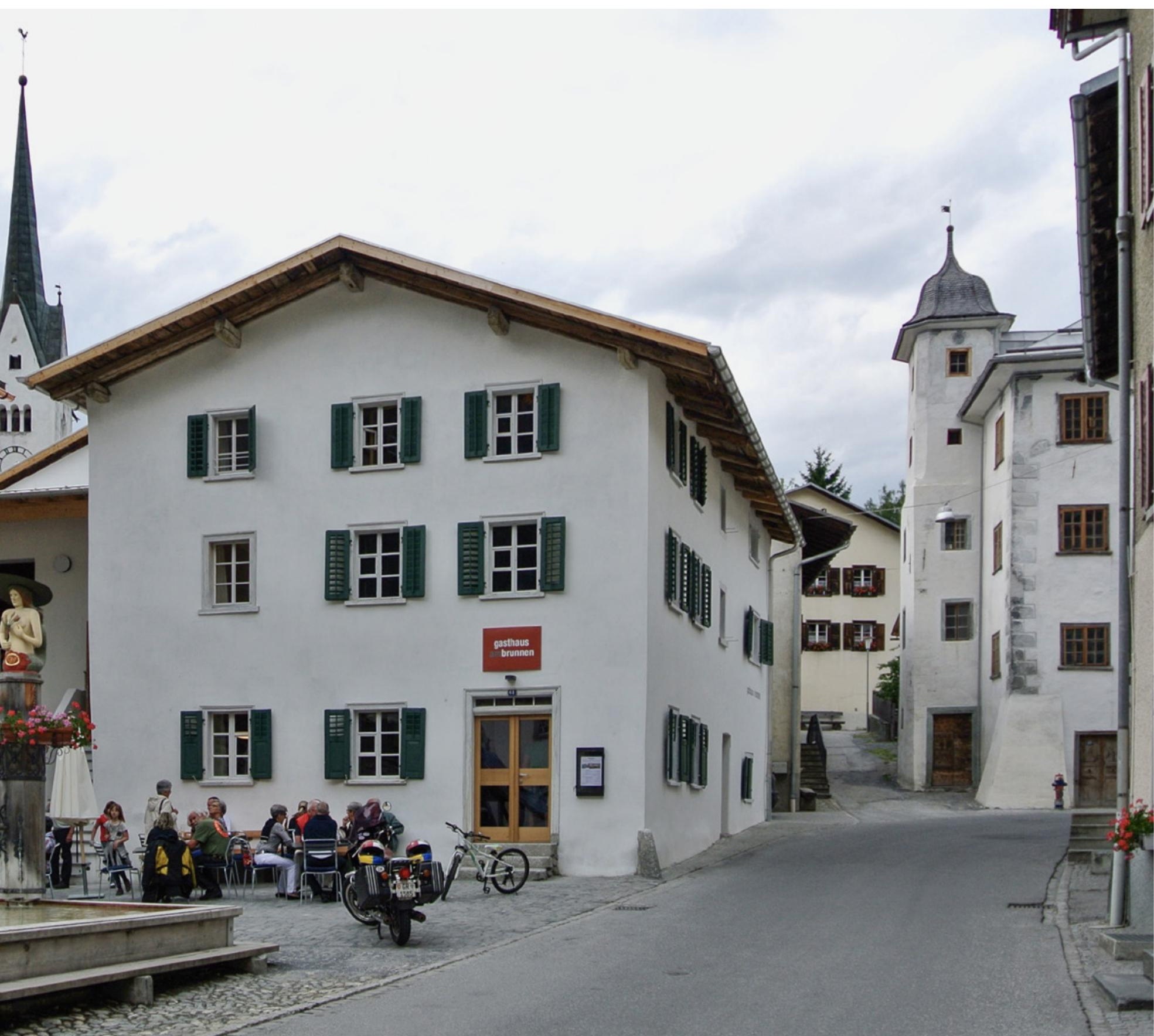


heizt. Die kalten Bereiche können auch als Antwort auf den Klimawandel verstanden werden.

\section{Individuelles Wohnen am Stadtrand}

Einen anderen Weg geht der Bündner Architekt Men Duri Arquint. Er entwickelte im Böschengut in Chur einen Wohnkomplex mit elf individuellen Wohneinheiten, die nach dem Prinzip «low rise, high density» einen grossen, naturbelassenen Zwischenraum aufspannen. Obwohl die einzelnen Volumina zusammenhängen, ist jede Einheit für sich so ausgerichtet, dass keine störenden Einsichten des Nachbars möglich sind und gelichzeitig jede Einheit Fenster und Blicke auf alle vier Seiten besitzt. Referenzen dafür waren sowohl die Siedlungen Kingohusene in Hillerød (1956-58) und diejenige in Fredensborg (1962-63). Zudem reagiert der Architekt auf die überzeugende Planung Böschengut 1 von Bearth und Deplazes, wo eine dreigeschossige Siedlungsstruktur gekonnt in die Hanglage gesetzt wurde oder der etwas weniger eleganten Überbauung Böschengut 2 von Richard Brosi. Arquint vertraut auf bewährte Konstruktionen und langlebige Materialisierungen wie
Aussenwände mit verputztem Einsteinmauerwerk, Platten wurden im Inneren ohne Fugen auf Knirsch verlegt oder der Dachabschluss ist nicht ein Blech, sondern ein Abschlussstein.

Auch in den Alpen muss mit dem Siedlungsraum sparsam umgegangen werden, das urbare Land ist knapp. Der kompakte Bau schafft Platz für einen durchgängigen Naturräum, der von Tieren als Weg auf die andere Talseite benutzt wird. Die Siedlung bildet nicht eine diffuse Ansammlung von Einzelbauten, sondern schafft ein Quartier mit klaren Freiräumen. Die Siedlung steht zudem auf städtischem Grund, der verpachtet wurde. Dies führt zu einem etwas tieferen Kaufpreis und ermöglicht der Stadt nach Ablauf der Pacht die Parzelle falls nötig anderweitig weiter zu entwickeln.

\section{Fortschritt im alpinen Raum}

Alpine Architektur besitzt Innovationskraft, kann mit neuen Lösungen Bestehendes stärken, aber auch Neues schaffen. Dadurch werden die Lebensgrundlagen in den oft wirtschaftlich prekären alpinen Situationen nachhaltig gestärkt. Stan-

Abb. 2

Theaterturm des

Kulturfestivals

Origen, Giovanni Netzer, Bauingenieur

Walter Bieler.

Julierpass $(\mathrm{CH})$, 2016 (foto Daniel A.

Walser).

Abb. 3

Siedlung Tusculum im Böschengut, Men

Duri Arquint. Chur

(CH), 2020 (foto

Daniel A. Walser).

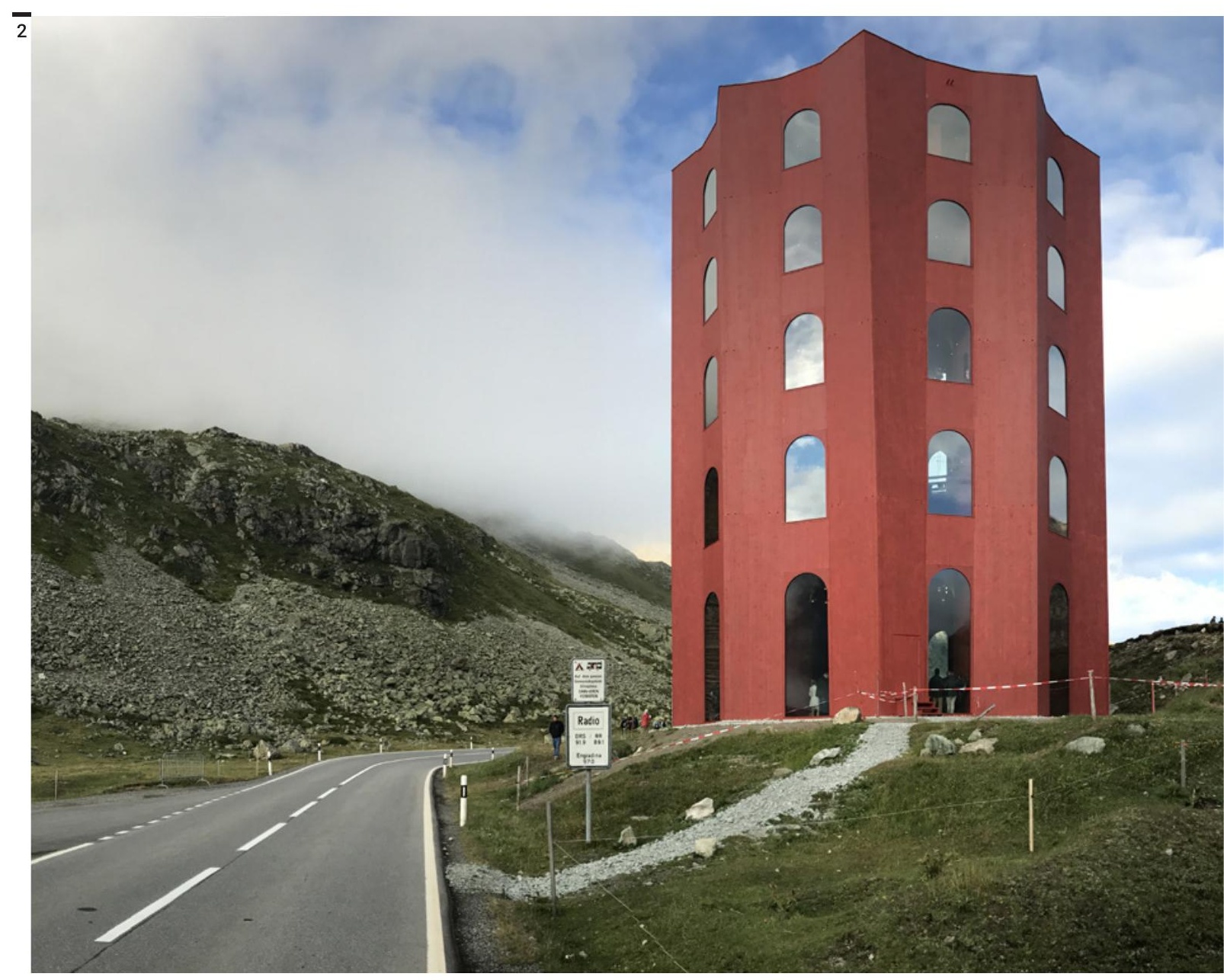



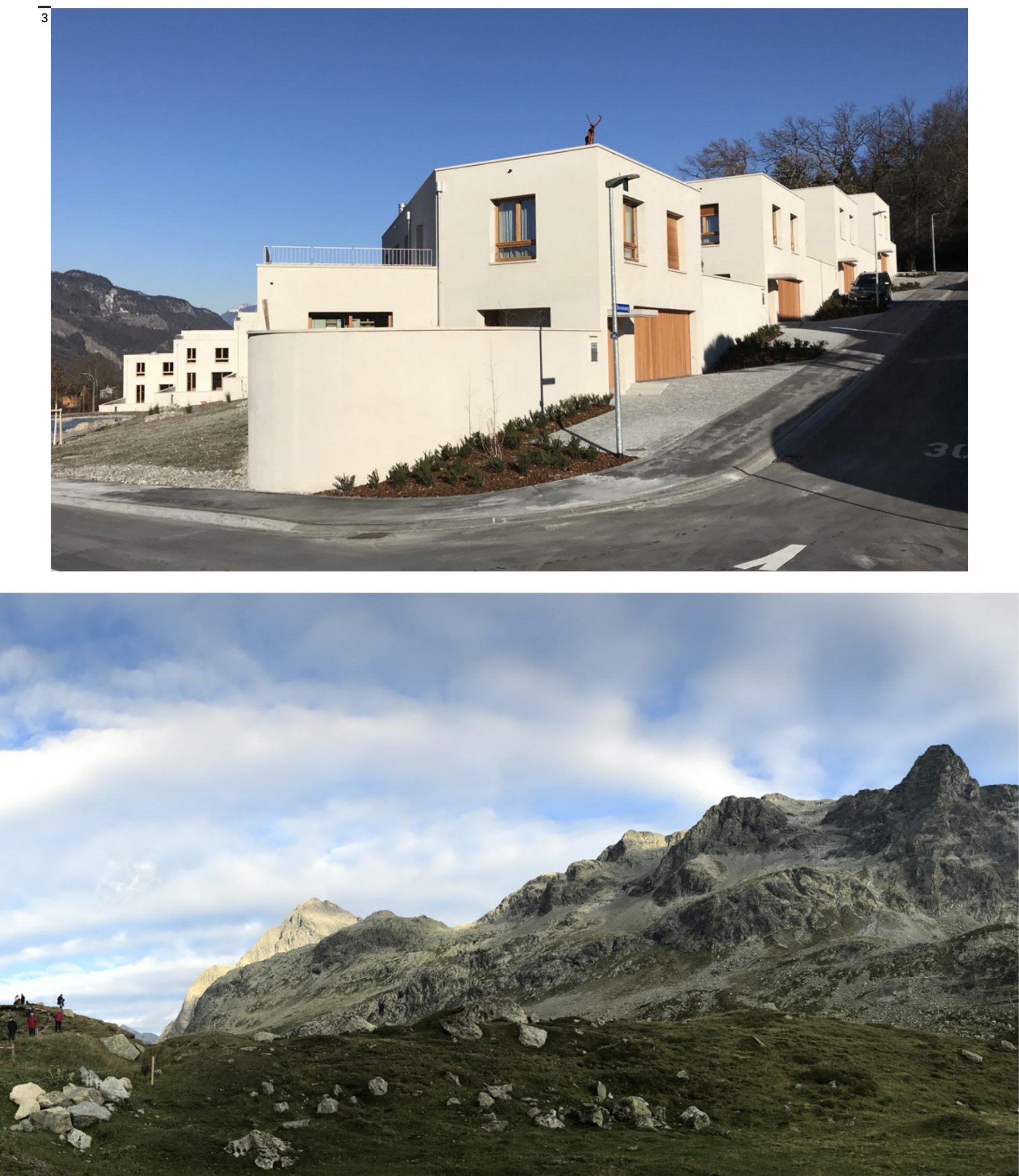
Abb. 4

Schulhaus und Kindergarten, Angela Deuber. Thal $(\mathrm{CH})$, 2014 (foto Daniel A. Walser).

Abb. 5

Schulhaus und

Kindergarten,

Raphael Zuber.

Grono (CH), 2011

(foto Javier Miguel

Verme)

Abb. 6-7

Kunstmuseum

Muzeum Susch,

Chasper Schmidlin und Lukas Voellmy.

Susch (CH) 2018 (foto Conradin Frei)

$\overline{4}$

$\overline{4}$

ś

5) $=5$

16.

$-2500010$

$0 \times 10$.

- $x$ sponn

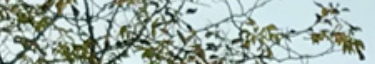

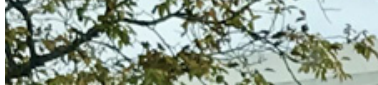

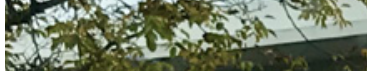

. 12

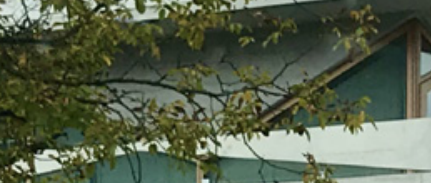

(4.)
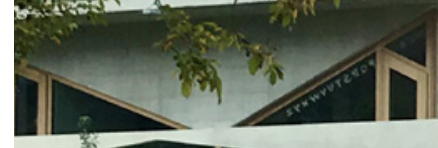

- 14,252

2
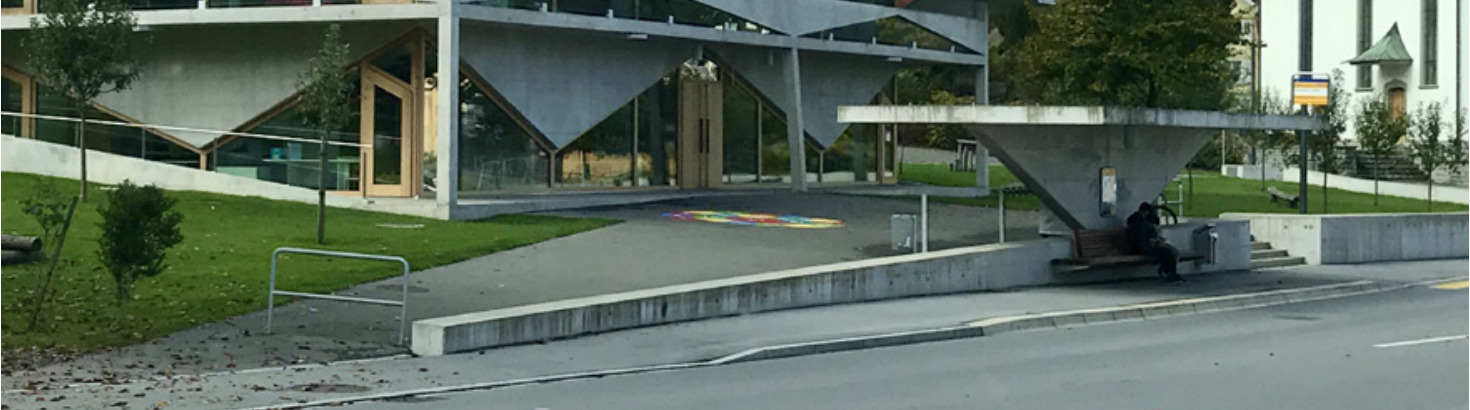

$\overline{5}$

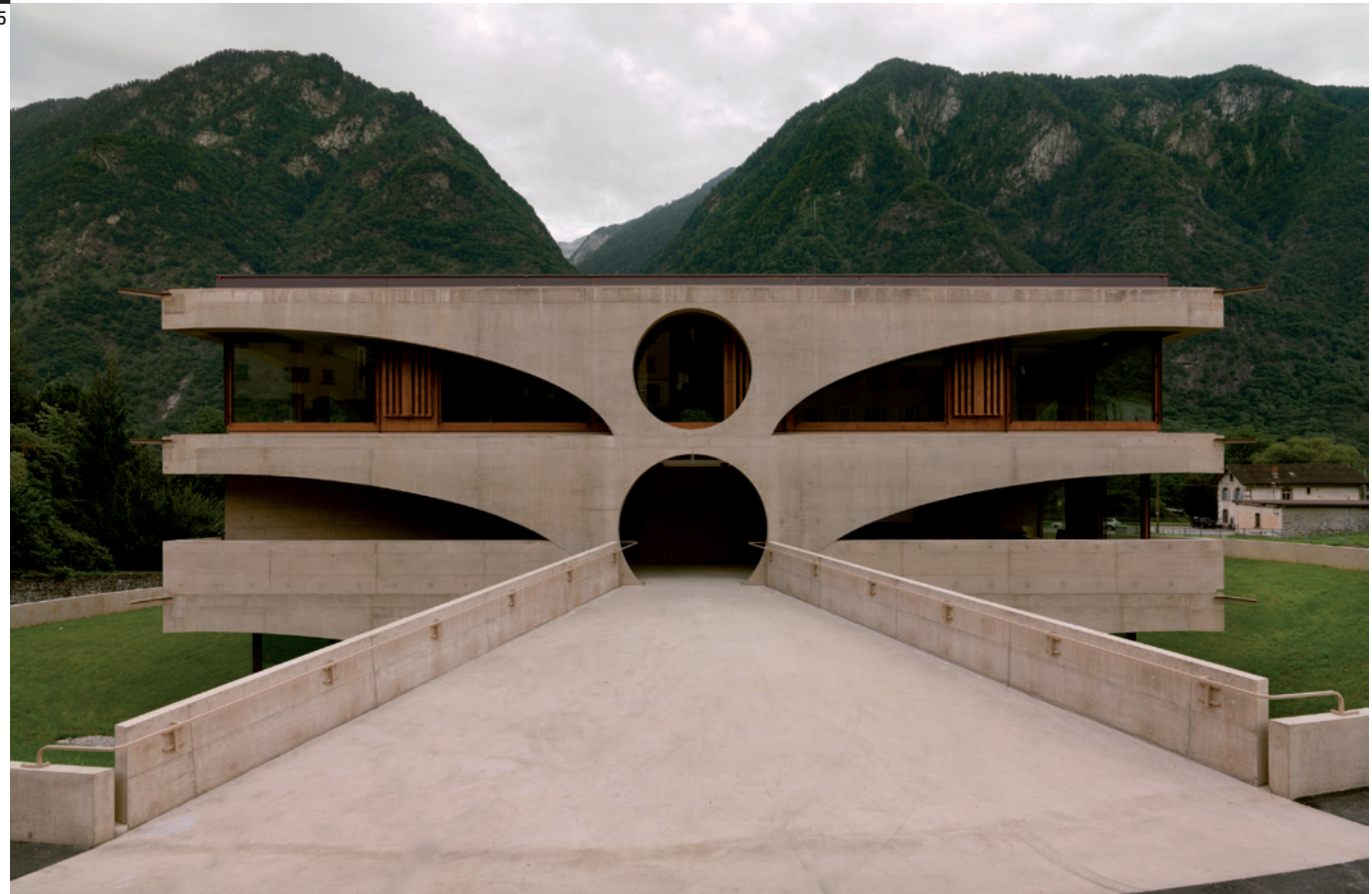




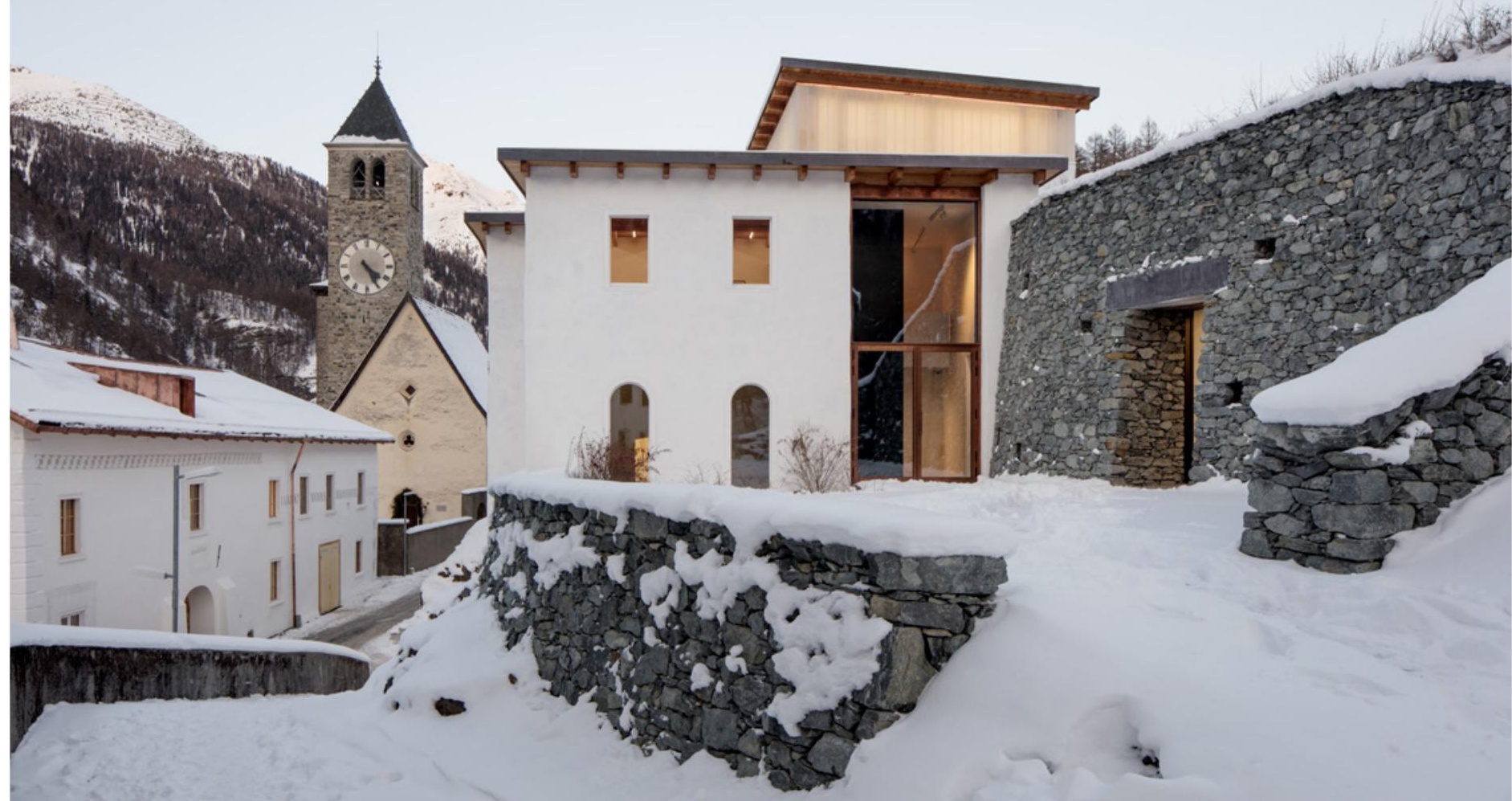

$\overline{7}$

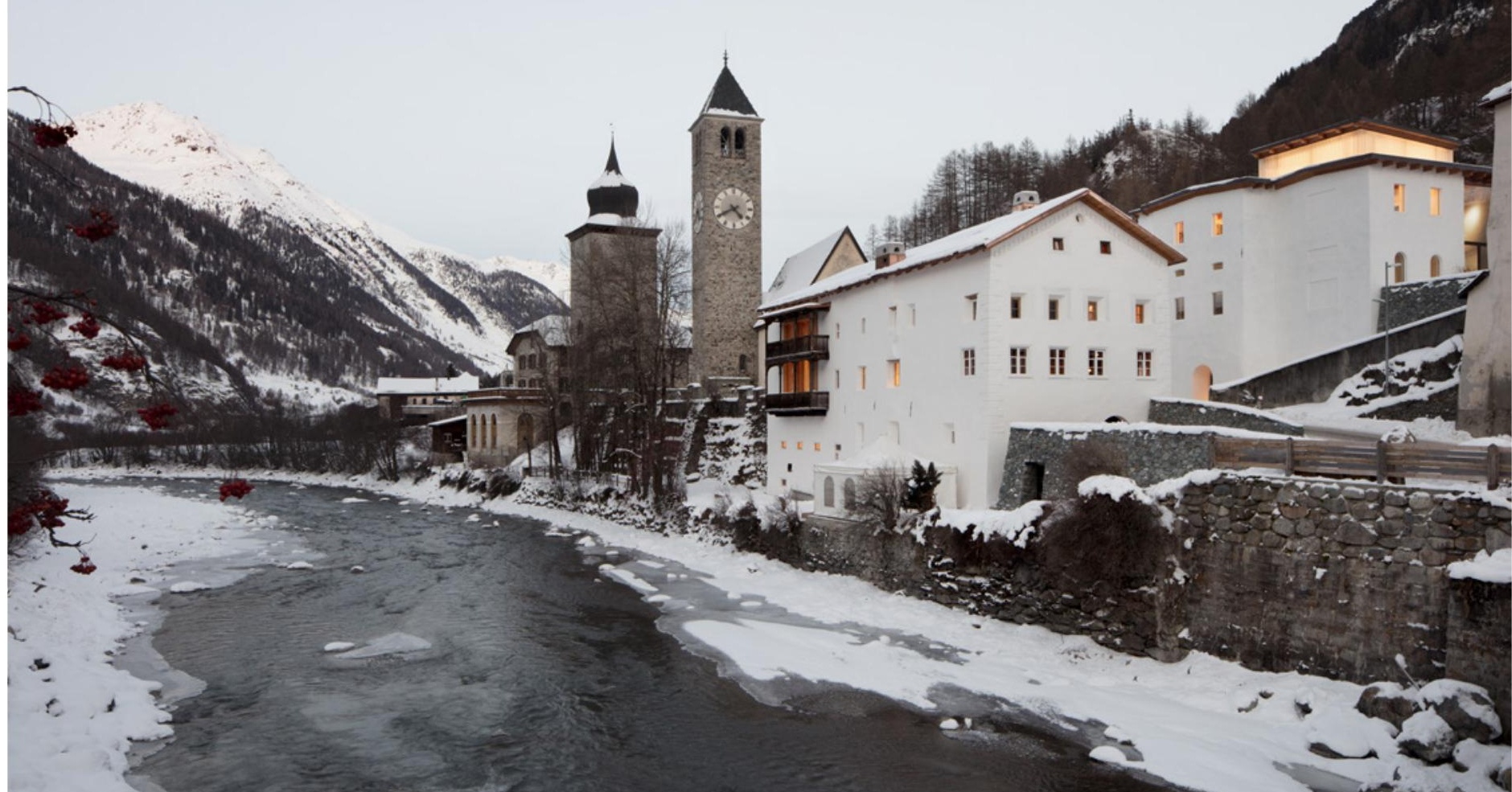


the

Nites

1

(3)

Sond

( ) 1

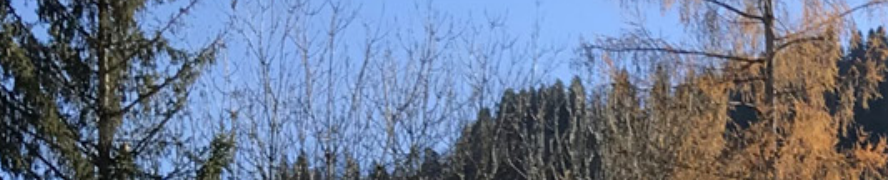

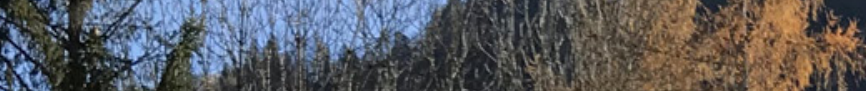

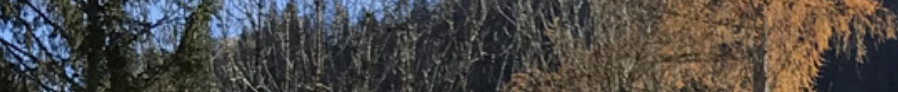

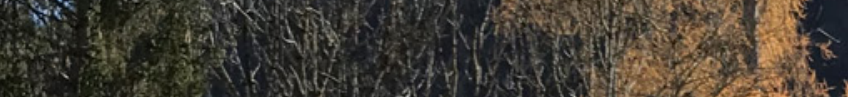

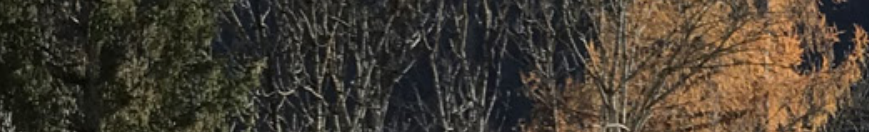

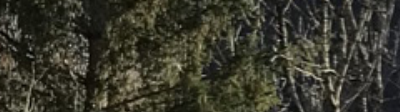

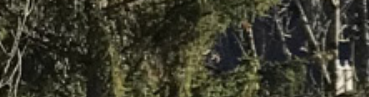

(2) 30 int

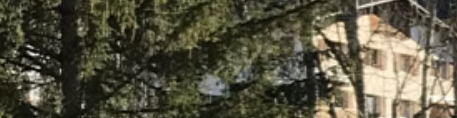

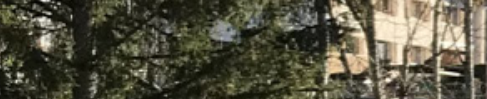

xis. -3x y

(4)

$4 n, y+2$

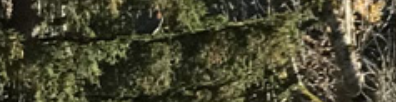

$+16)^{2}$

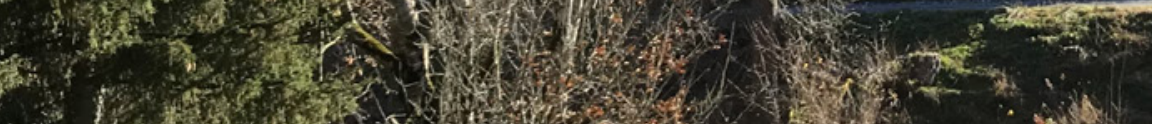

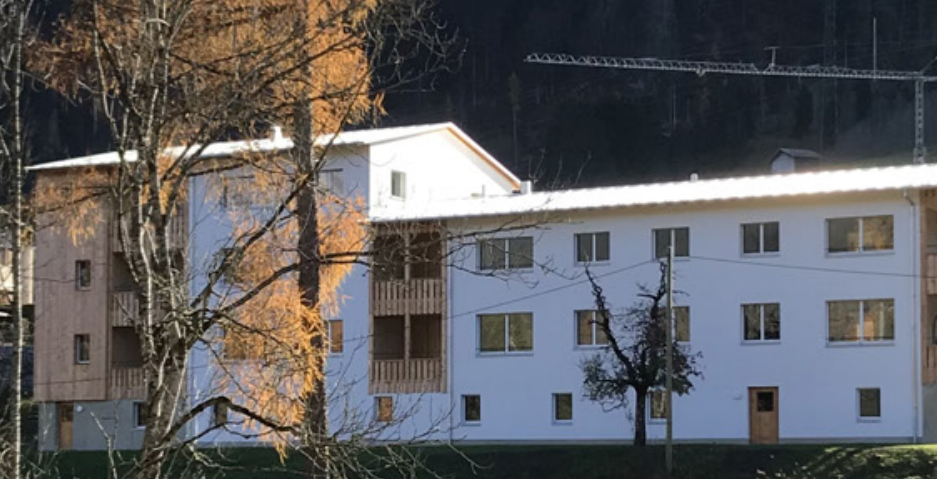

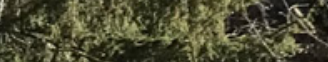

W

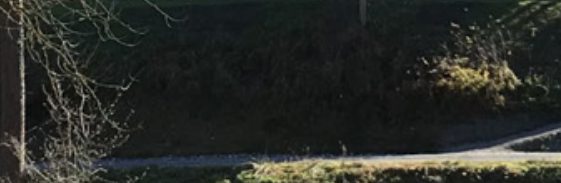

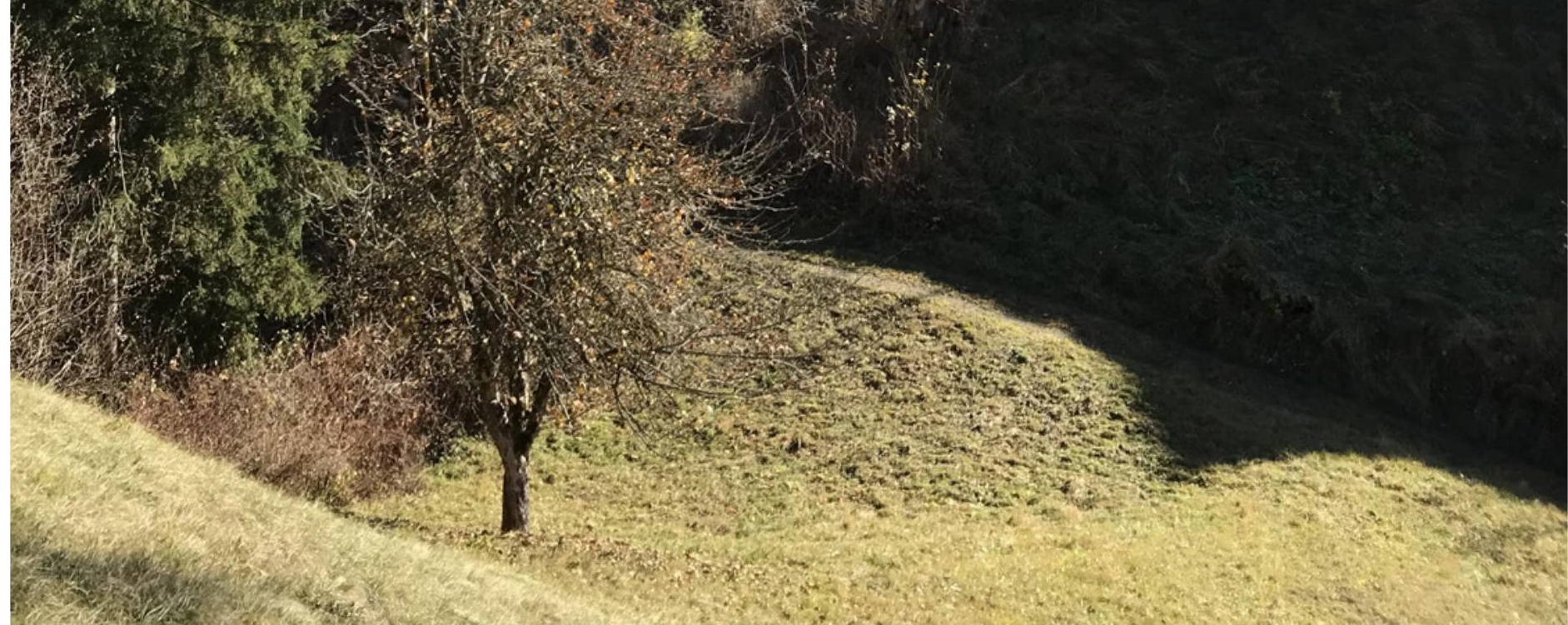

